\section{Romberg Test}

John E. Mendoza

Department of Psychiatry and Neuroscience, Tulane Medical School and SE Louisiana Veterans Healthcare System, New Orleans, LA, USA

\section{Definition}

A standard maneuver in a neurological exam designed to assess mechanisms mediating balance which involves having the patient stand with feet together and eyes closed.

\section{Current Knowledge}

Balance or equilibrium when standing is primarily mediated by the cerebellum. However, to maintain balance, the cerebellum needs sensory input from vision, the vestibular system, and/or proprioceptive feedback from the lower extremities. In theory, when intact, the cerebellum can maintain postural stability if at least two of these three sensory systems are functioning properly, although disruption of the vestibular system alone can potentially result in significant unsteadiness. Once it has been established that the patient can maintain balance by standing with the feet together and the eyes open (a test of the integrity of the vermis of the cerebellum), the patient is asked to maintain the same stance but now with the eyes closed. If the patient has marked difficulty in maintaining balance under this latter condition, the results are considered pathological and are referred to as exhibiting a "positive Romberg."

Following Romberg's original hypothesis that this was a specific indication for syphilis or tabes dorsalis (a disease affecting the posterior columns), most texts suggest that a positive Romberg is a sign of posterior column disease (loss of two of three sensory systems, namely, vision and proprioception). However, two other possibilities need to be considered. Certain peripheral neuropathies that might disrupt the transmission of proprioceptive information to the posterior columns via the dorsal nerve roots can produce similar effects. Subtle vestibular system deficits, which might not be sufficient to significantly disrupt balance on their own, might become clinically symptomatic when also deprived of visual feedback, but such deficits are usually either more paroxysmal or likely to be related to certain movements of the head (benign positional vertigo).

\section{Cross-References}

Vestibular System

\section{References and Readings}

Pearce, J. M. S. (2005). Romberg and his sign. European Neurology, 53, 210-213. 